\title{
O USO DE REDES NEURAIS ARTIFICIAIS PARA ANÁLISE DE DADOS DE RAIOS cósmicos
}

\section{APPLICATION OF ARTIFICIAL NEURAL NETWORKS FOR ANALYSIS OF COSMIC RAY DATA}

\author{
Alessandro Gerson Moura Izzo de Oliveira' \\ Marlos Rockenbach ${ }^{2}$
}

RESUMO: A detecção das partículas que compõem os raios cósmicos galácticos pode fornecer importantes dados acerca do meio interplanetário, auxiliando muito na compreensão dos fenômenos do Clima Espacial. A acurácia dessa informação, no entanto, está diretamente correlacionada à nossa capacidade de interpretá-la. Utilizamos assim redes neurais dos modelos MLP (Multilayer Perceptron) e NARX (Nonlinear AutoRegressive with eXogenous inputs) para tentar simular a densidade de raios cósmicos no meio interplanetário, utilizando como vetor de entrada da rede dados do campo magnético interplanetário (Interplanetary Magnetic Field - IMF) obtidos do satélite ACE (Advanced Composotion Explorer) que está no ponto Lagrangiano (L1). A análise do modelo, arquitetura e características das redes que obtiveram maior sucesso de simulação podem não só indicar a melhor maneira de adequar esse recurso computacional à tarefa, mas também trazer indícios valiosos da relação entre o IMF e os raios cósmicos.

Palavras-chave: raios cósmicos; heliosfera; redes neurais artificiais.

ABSTRACT: The analysis of particles that compose the galactic cosmic rays produce important data about the conditions of the interplanetary environment, improving the understanding of the Space Weather phenomena. The precision of this data, however, is directly correlated with our capacity to understand them. We used Multi-Layer Perceptrons model (MLP) and Nonlinear AutoRegressive with eXogenous inputs (NARX) models of Artificial Neural Networks (ANN) to simulate the density of cosmic rays in the interplanetary medium, applying input vector data from the Advanced Composition Explorer (ACE) satellite located in the Lagrangian point (L1) about the Interplanetary Magnetic Field (IMF). The analysis of the model, topology, and characteristics of the most successful ANNs may not only indicated the best way to adjust this computational tool but also provide valuable clues about the relation between the galactic cosmic rays and the IMF data.

Keywords: cosmic rays; heliosphere; artificial neural network.

\footnotetext{
1 Divisão de Astrofísica - Instituto Nacional de Pesquisas Espacias - INPE. E-mail: a.ger@aol.com.

2 Docente da Universidade do Vale do Paraíba - Univap / Instituto de Pesquisa e Desenvolvimento - IP\&D - Laboratório de Física e Astronomia. E-mail: marlos@univap.br.

Revista Univap - Edição Especial - revista.univap.br

São José dos Campos-SP-Brasil, v. 19, n. 34, nov.2013. ISSN 2237-1753
} 


\section{INTRODUÇÃO}

Este texto apresenta, de forma sucinta, o trabalho de investigação de correlação entre as variáveis concernentes ao meio interplanetário e dados de raios cósmicos obtidos por meio de detectores de solo, mais especificamente os detectores que compõem a Rede Global de Detectores de Múons (Global Muon Detector Network GMDN). A investigação, que tem lugar no Instituto de Pesquisa e Desenvolvimento (IP\&D) da Universidade do Vale do Paraíba (UNIVAP), visa à ampliar a compreensão do transporte de raios cósmicos pela heliosfera por meio de um instrumental computacional capaz de produzir métodos de simulação e predição de variáveis: as redes neurais artificiais (Artificial Neural Networks - ANN).

Nesse contexto, por meio das ANNs, produzem-se simulações da densidade de raios cósmicos, no meio interplanetário, baseadas nas componentes do vetor IMF dado no sistema de coordenadas GSE (Geocentric Solar Ecliptic) e simulações da intensidade do IMF, baseados nos componentes do vetor anisotropia dos raios cósmicos durante períodos de grande atividade geomagnética (ECHER et al., 2008). A acurácia das simulações e as estruturas e métodos empregados na ANN são indicativos das características da relação que existem entre as grandezas. É importante salientar que o emprego de ANNs para interpretação de dados espaciais é bastante novo, tornando consideravelmente árduo os esforços de investigação; por outro lado, os resultados obtidos são inéditos e muito profícuos.

\section{DADOS E METODOLOGIA}

O método estabelecido por
Rockenbach et al. (2011) permite inferir a densidade de partículas de raios cósmicos no meio interplanetário e as componentes do vetor anisotropia de incidência dessas partículas na Terra, com base em dados obtidos da GMDN. Por sua vez, o satélite ACE, localizado no ponto Lagrangiano (L1) entre a Terra e o Sol, fornece dados in situ das variáveis do meio interplanetário, como o módulo IMF, intensidade e direção das componentes do vetor IMF, além de dados de diversos outros elementos do plasma do meio interplanetário.

A origem do método computacional, proposto pelas redes ANNs, remonta os esforços de compreender o funcionamento das redes neurais naturais empreendidos em meados de 1950 (KOVÁcS, 2006). ANNs são capazes de categorizar e/ou produzir estimativas baseadas em um processo prévio de treinamento. 0 treinamento ou aprendizado da rede não é, no entanto, um procedimento trivial. Quando o processo de treinamento é supervisionado, as dificuldades principiam já na escolha, divisão e tratamento dos dados que irão compor o conjunto de treinamento, tanto no vetor de dados de entrada quanto no vetor de dados de resposta esperada. A topologia, ou seja, a quantidade e posição dos nodos da rede e a seleção do algoritmo de treinamento apropriado para produzir uma rede otimamente treinadas são outras dificuldades inerentes à preparação de ANNs otimamente treinadas. Redes otimamente treinadas são ANNs treinadas de forma a contar com ampla capacidade de generalização, sendo pouco suscetíveis a ruídos estatísticos nos dados.

Embora inicialmente tenham sido adotadas redes do tipo MLP, os estudos preliminares (IZZO DE OLIVEIRA, 2013) 
mostraram que redes do tipo NARX treinadas pelo algoritmo de LevenbergMaquardt produzem as simulações mais precisas. A estrutura topológica da ANN é usualmente, determinada, empiricamente, pelo método de tentativa e erro (TAGLIAFERRI et al., 2003); contudo, a estrutura topológica com 5 nodos na primeira camada oculta e 20 nodos na segunda camada oculta mostrou-se consistentemente um bom ponto de partida.

É importante notar que redes NARX são redes muito similares a redes MLP, diferenciando-se pelo fato de serem retroalimentadas. Isso significa que dados referentes ao vetor de saída de iterações anteriores da rede integram o vetor de entrada em iterações posteriores armazenados na rede por meio de operadores de atraso. Isso pode ser feito de dois modos distintos: (1) no modo sérieparalelo, o vetor de entrada é alimentado com dados observados da grandeza de saída. Nesse modo, a rede não é, de fato, retroalimentada, uma vez que não produziu o dado utilizado no vetor de entrada e fica limitada à simulação de curto termo, estimando valores apenas uma única iteração adiante da atual. (2) No modo paralelo, o valor de saída que passa a integrar o vetor de entrada é aquele estimado pela rede em iterações anteriores. Embora menos preciso que o modo sérieparalelo, esse modo permite estimativas várias iterações adiante do presente momento, produzindo simulações de longo termo.

Simulações de ambos os modos são produzidas, usualmente uma rede que obteve um resultado consideravelmente bom, quando aplicada no modo paralelo, produzirá simulações ainda mais precisas quando utilizada no modo série-paralelo.

A qualidade das simulações produzida é avaliada por um algoritmo computacional que determina a correlação entre a variação da grandeza simulada pela ANN e a variação da grandeza observada, bem como o desvio padrão do conjunto dado pelo valor da diferença entre 0 ponto simulado e o valor, de fato, observado.

\section{RESULTADOS}

As simulações de duas redes NARX distintas estão ilustradas a seguir. O primeiro par de simulações é referente a uma rede NARX que simulou os valores da intensidade de raios cósmicos, baseada em dados observados do vetor IMF. Na primeira simulação a rede NARX, foi utilizado no modo paralelo, enquanto, na segunda, adotou-se o modo série paralelo.

A Figura 1 mostra a variação das componentes do vetor IMF, utilizadas como vetor de entrada para a primeira rede NARX. O período de 09 de Abril de 2001 a 14 de Abril de 2001 (período de Abril de 2001), foi utilizado durante o processo de treinamento da ANN, porém a simulação foi realizada para o período de 12 de Dezembro de 2006 a 18 de Dezembro de 2006 (período de Dezembro de 2006), de forma a garantir resultados de uma rede com boa capacidade de generalização. 


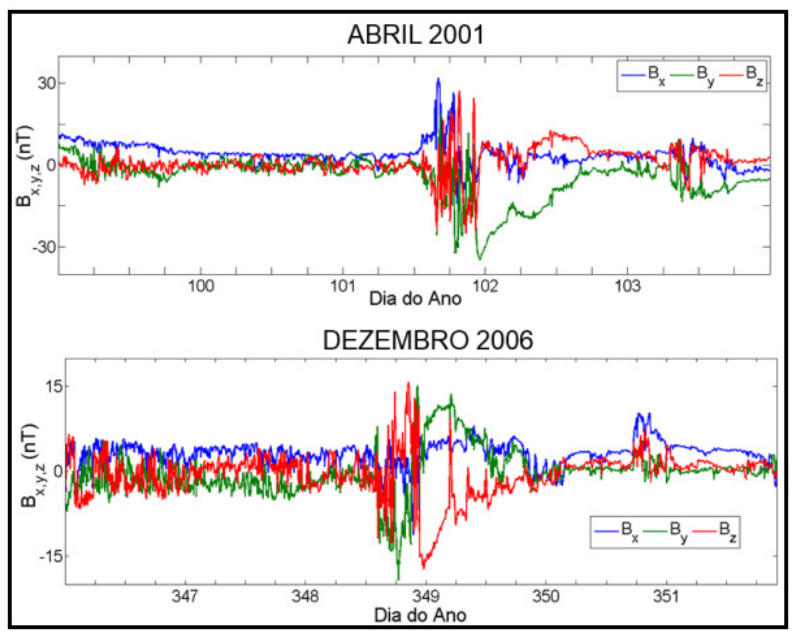

Figura 1 - Dados de entrada da primeira rede NARX. O primeiro painel, a partir de cima, mostra a variação das componentes do vetor IMF, no período de abril de 2001, como registrado pelo satélite ACE. Os dados do período de abril de 2001 foram empregados para treinamento da rede. O painel de baixo mostra a variação das componentes durante o período de dezembro de 2006. A rede NARX utilizou esses dados para produzir as simulações da densidade de raios cósmicos. Em ambos painéis em azul a componente $B_{x}$ do IMF no sistema GSE, em verde a componente $B_{y}$ e em verde a componente $B_{z}$.

É importante notar o quão distinto foi o comportamento de cada uma das três componentes do vetor IMF para cada período, para que se possa avaliar a qualidade das simulações obtidas.

A Figura 2 mostra a simulação de longo termo, produzida pela rede NARX no modo paralelo.

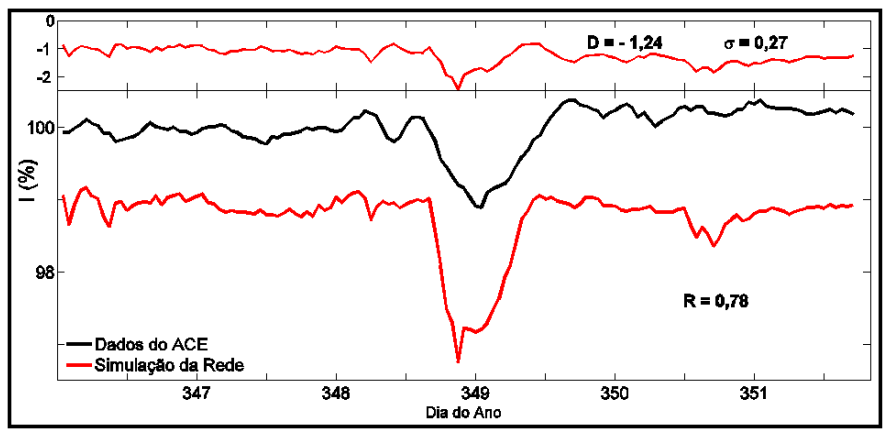

Figura 2 - Simulação da densidade de raios cósmicos no período de dezembro de 2006. 0 primeiro painel, a partir de cima, mostra as diferenças, ponto-a-ponto, entre a simulação e os dados observados. $D$ indica a diferença média e $\sigma$ o desvio padrão das diferenças. No painel de baixo, o traço preto mostra a variação percentual da densidade de raios cósmicos, como determinada por Rockenbach et al. (2011) e o traço vermelho indica a simulação de longo termo, realizada pela rede NARX. R refere-se ao coeficiente de correlação entre a simulação e os dados observados.

É possível notar que simulação desviou sistematicamente dos valores esperados, mas que o principal decréscimo ficou razoavelmente bem representado.
A Figura 3 mostra a simulação da mesma rede, porém, agora, utilizada no modo sérieparalelo. É possível notar uma significativa melhora em todos os aspectos da simulação. 


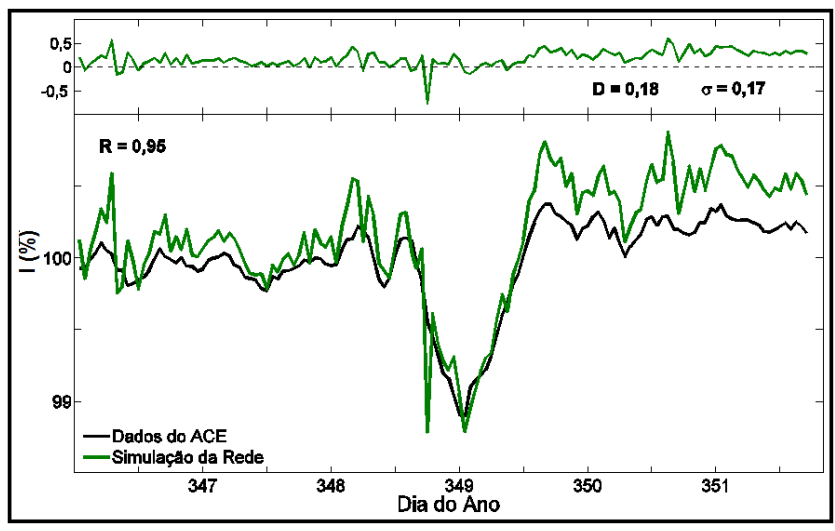

Figura 3 - Simulação da densidade de raios cósmicos no período de dezembro de 2006.0 primeiro painel, a partir de cima, mostra as diferenças, ponto-a-ponto, entre a simulação e os dados observados. $D$ indica a diferença média e $\sigma 0$ desvio padrão das diferenças. No painel de baixo, o traço preto mostra a variação percentual da densidade de raios cósmicos, como determinada por Rockenbach et al. (2011) e o traço verde indica a simulação de curto termo, realizada pela rede NARX. R refere-se ao coeficiente de correlação entre a simulação e os dados observados.

\section{O segundo par de simulações é} proveniente de uma rede NARX que simulou a intensidade do IMF, tendo como dados do vetor de entrada as componentes do vetor anisotropia dos raios cósmicos.
$\mathrm{Na}$ Figura 4, a variação dos dados do vetor anisotropia para os períodos de abril de 2001 e dezembro de 2006. Mais uma vez os dados de abril de 2001 foram utilizados para treinamento enquanto a simulação foi realizada com dados de dezembro de 2006.

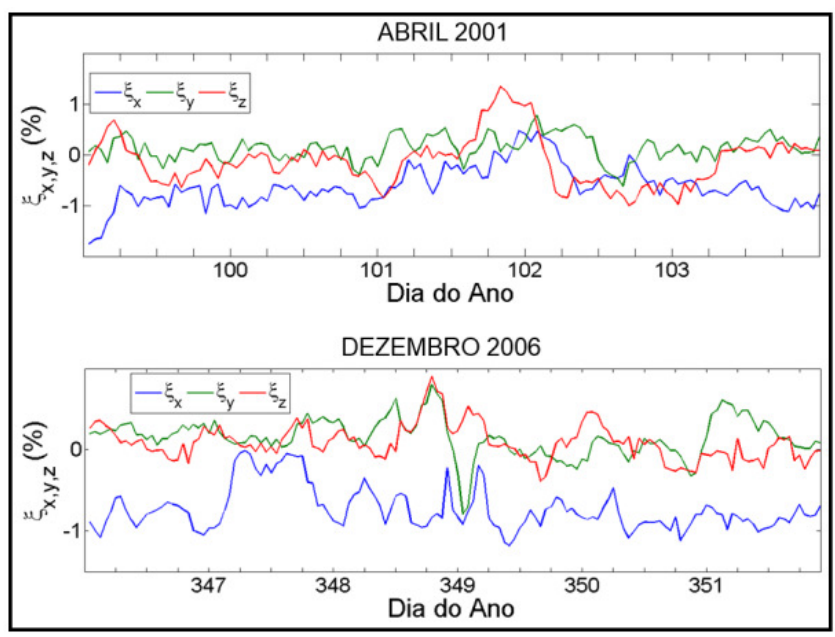

Figura 4 - 0 primeiro painel, a partir de cima, mostra a variação das componentes do vetor anisotropia dos raios cósmicos no período de abril de 2001, como calculada por Rockenbach et al. (2011). Os dados do período de abril de 2001 foram empregados para treinamento da rede. $O$ segundo painel mostra a variação das componentes durante o período de dezembro de 2006. A rede NARX, treinada com período de abril de 2001, utilizou esses dados para produzir as simulações da intensidade do IMF. Em ambos painéis em azul a componente $\xi_{x}$ do IMF no sistema GSE, em verde a componente $\xi_{y}$ e em verde a componente $\xi_{z}$. 
Dessa vez, como mostra a Figura 5, mesmo a simulação do modo paralelo foi bastante correlata $e$ os valores se mantiveram consideravelmente bem nas vizinhanças dos valores esperados. No entanto, um decréscimo inesperado ocorre na simulação por volta do dia 347 e o pico que ocorreu na metade do dia 350 não foi reproduzido pela simulação.

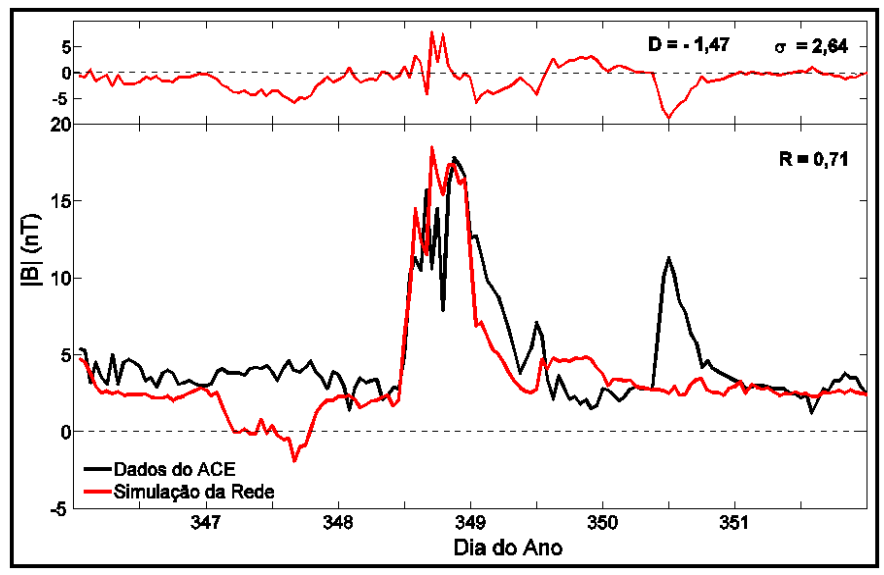

Figura 5 - Simulação da intensidade do IMF, no período de dezembro de 2006. 0 primeiro painel, a partir de cima, mostra as diferenças, ponto-a-ponto, entre a simulação e os dados observados. $D$ indica a diferença média e $\sigma$ o desvio padrão das diferenças. No segundo painel, o traço preto mostra a variação da intensidade do IMF, como observada pelo satélite ACE e o traço vermelho indica a simulação de longo termo, realizada pela rede NARX. R referese ao coeficiente de correlação entre a simulação e os dados do ACE.

Quando utilizada no modo série- produz indicadores de qualidade melhores, paralelo, a simulação de curto termo como mostra a Figura 6.

produzida pela rede NARX novamente

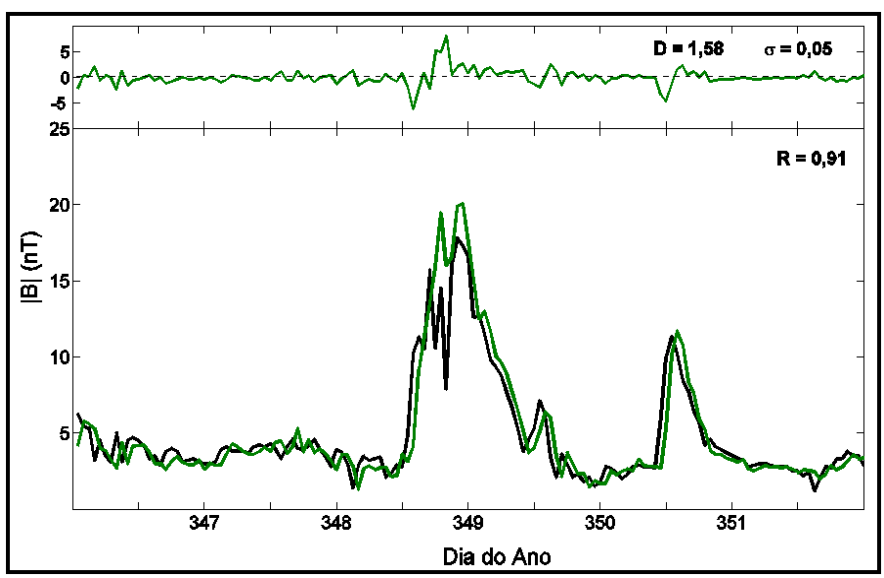

Figura 6 - Simulação da intensidade do IMF no período de dezembro de 2006. O primeiro painel, a partir de cima, mostra as diferenças, ponto-a-ponto, entre a simulação e os dados observados. $D$ indica a diferença média e $\sigma$ o desvio padrão das diferenças. No segundo painel, o traço preto mostra a variação da intensidade do IMF, como observada pelo satélite ACE e o traço vermelho indica a simulação de longo termo, realizada pela rede NARX. $R$ referese ao coeficiente de correlação entre a simulação e os dados do ACE. 


\section{CONCLUSÕES}

Os resultados corroboram a ideia de que as ANNs são ferramentas computacionais poderosas e bastante promissoras quando empregadas a ciências espaciais. Aprimoramento nas características do conjunto de treinamento provavelmente produziriam redes mais acuradas. Mantenha-se em mente que 0 treinamento das simulações aqui apresentadas baseou-se em um vetor de dados composto por uma única grandeza. Incrementar o vetor de entrada de dados com duas ou mais grandezas é uma interessante possibilidade para melhorar a qualidade das simulações.

É de fato muito interessante uma abordagem na qual as ANNs atuem como instrumentos de previsão para elementos componentes do Clima Espacial, além disso, quando utilizadas para simular o IMF com base nos dados fornecidos pelos detectores da GMDN, as ANNs produzem a possibilidade de estimar dados interplanetários com base em dados observados na superfície da Terra, constituindo-se, assim, de mais um método alternativo a tecnologia espacial.

De qualquer modo, os resultados indicam um campo de pesquisa profícuo que merece maior atenção por parte dos autores das pesquisas espaciais.

\section{REFERÊNCIAS}

ECHER, E. et al. Interplanetary conditions causing intense geomagnetic storms (Dst $\geq 100 \mathrm{nT}$ ) during solar cycle 23 (19962006). Journal of Geophysical Research, v. 113, 2008. doi:10.1029/2007JA012744.

IZZO DE OLIVEIRA, A. G. Estudo da Relação entre o Campo Magnético Interplanetário e a Intensidade de Raios Cósmicos via Redes Neurais. 2013. Dissertação (Mestrado em Física e Astronomia) - Instituto de Pesquisa e Desenvolvimento. Universidade do Vale do Paraíba, São José dos Campos, 2013.

KOVÁCS, Z. L. Redes Neurais Artificiais: Fundamentos e Aplicações: Um texto básico, 4. ed. São Paulo: Livraria da Física, 2006.

ROCKENBACH, M. et al. Geomagnetic storm's precursors observed from 2001 to 2007 with the Global Muon Detector Network (GMDN), Geophysical Research Letters, v. 38, 2011. doi:10.1029/2011GL048556.

TAGLIAFERRI, R. G. et al. A. Neural networks in astronomy. Neural Netw., v. 16, n. 3-4, p. 297-319, Apr-May 2003. doi:10.1016/s0893-6080(03)00028-5. 